\title{
Comportamento visual e perfil socioeconômico e demográfico de recém-nascidos prematuros da Maternidade do Hospital das Clínicas de Pernambuco - UFPE
}

\author{
Visual behavior and socioeconomic and demographic profile of the premature newborns from \\ Federal University of Pernambuco Maternity (UFPE)
}

Nathalia Christina Monteiro de Souza ${ }^{1}$, Ana Cláudia Vasconcelos Martins de Souza lima², Heloisa Gagheggi Ravanini Gardon Gagliardo ${ }^{3}$, Raquel Costa Albuquerque ${ }^{2}$, Tatiana Celentano Cardoso², Flávia Regina Ribeiro Cavalcanti² ${ }^{2}$ Rodrigo Evaldo de Azevedo Coêllho ${ }^{4}$

\section{RESUMO}

Objetivo: Caracterizar o comportamento visual e perfil socioeconômico e demográfico de recém-nascidos prematuros no primeiro mês de idade cronológica. Métodos: Estudo descritivo de corte transversal, aninhado a um estudo longitudinal intitulado: "Comparação do comportamento visual no primeiro trimestre de vida de lactentes nascidos pré-termo em duas maternidades da cidade do Recife/PE". A amostra foi composta por 52 recém-nascidos prematuros recrutados de junho de 2007 a junho de 2008, na Maternidade do Hospital das Clínicas da Universidade Federal de Pernambuco. Foram coletados dados biológicos, socioeconômicos e demográficos dos recém-nascidos e famílias, por meio de consulta aos prontuários e entrevista com as genitoras. Para caracterizar o comportamento visual dos recémnascidos utilizou-se o Método de Avaliação do Comportamento Visual de Lactentes. Resultados: Observou-se que a maioria dos recém-nascidos era do sexo masculino, com idade gestacional entre 33 semanas e 36 semanas e 6 dias, apresentaram comportamento visuomotor adequado para a faixa etária pesquisada e que a maioria das famílias apresentava boas condições socioeconômicas e demográficas. Ainda fo possível detectar em $19 \%$ da amostra sinais oculares, os quais foram encaminhados para um Serviço de Oftalmologia.

Conclusões: Os resultados apontam o método como um instrumento importante na triagem visual, possibilitando a detecção oportuna de alterações visuais em lactentes prematuros desde o $1^{\circ}$ mês de vida. O olhar clínico da avaliação terapêutica ocupacional de lactentes deve voltar-se para a observação do comportamento considerando não apenas os riscos biológicos, mas também a influência que os fatores ambientais e sociais exercem sobre o desempenho funcional da criança.

Descritores: Prematuro; Triagem neonatal; Visão ocular; Fatores socioeconômicos; Fatores biológicos

\begin{abstract}
Objective: To characterize the behavior of premature newborns in the first year of chronological age.

Methods: This is a cross-sectional descriptive study, bound to a longitudinal study titled: "Comparison of visual behavior on the first quarter of year of life of premature nursling born at two maternities of Recife/PE." The sample was composed by 52 premature newborns selected from June, 2007 to June, 2008 from the Maternity of the Federal University of Pernambuco (UFPE). Biological, socioeconomic and demographic data was collected through medical records and interviews with progeny. Newborns were evaluated by the Assessment Guide of Visual Ability in Infants.

Results: Most of the newborns were male at a gestational period between 33 weeks and 36 weeks and 6 days, showed a good visual behavior development for the age researched, and most of the families showed good socioeconomical and demographic profile. Besides, it was possible to detect ocular signs in 19\% of sample, that were referred to an Ophthalmology Service.

Conclusion: This study results point out the method like an important key in the early detection and visual screening for premature nursling since the first month of life and it led us to believe that clinical view for occupational therapy intervention must be focused not only on biological risks but also at the influence environment in newborn performance.
\end{abstract}

Keywords: Infant, premature; Neonatasl screening; Vision, ocular; Socioeconomic factors; Biological factors
Estudo desenvolvido na Maternidade do Hospital das Clínicas da Universidade Federal de Pernambuco - UFPE - Recife (PE), Brasil.

${ }^{1}$ Terapeuta Ocupacional, Serviço de Reabilitação Profissional, Instituto Nacional de Seguridade Social - INSS - Natal (RN), Brasil.

2 Terapeuta Ocupacional, Departamento de Terapia Ocupacional, Universidade Federal de Pernambuco - UFPE - Recife (PE), Brasil.

${ }^{3}$ Terapeuta Ocupacional, Universidade Estadual de Campinas - UNICAMP - Campinas (SP), Brasil. ${ }^{4}$ Médico, Instituto de Olhos Potiguar; Hospital da Visão - Natal (RN), Brasil.

Endereço para correspondência: Nathalia Christina Monteiro de Souza. Rua José Felix Damasceno, 287 - Jaboatão dos Guararapes (PE) - CEP 54400-340

E-mail: natyufpe@yahoo.com.br

Recebido para publicação em 16.08.2008

Última versão recebida em 05.11 .2010

Aprovação em 16.12.2010

Nota Editorial: Depois de concluída a análise do artigo sob sigilo editorial e com a anuência do Dr. Pedro Carricondo sobre a divulgação de seu nome como revisor, agradecemos sua participação neste processo.

\section{INTRODUÇÃO}

m famílias de nível socioeconômico baixo há maior prevalên- cia de nascimentos prematuros, e a condição de prematuri- dade torna estas crianças mais vulneráveis aos efeitos do ambiente desfavorável do que as nascidas a termo ${ }^{(1)}$. A estimulação provinda do ambiente é a variável de maior impacto sobre o desenvolvimento infantil, existindo uma gama de associações entre a qualidade do ambiente, os fatores de risco biológicos e psicossociais ${ }^{(2)}$.

Crianças pré-termo, devido às privações sensoriais advindas do extenso período de internação apresentam maior incidência de atraso no desenvolvimento neuropsicomotor (DNPM) ${ }^{(3-5)}$.

Segundo a American Academy of Ophthalmology (AAO) $)^{(6)}$, a prematuridade é o maior fator de risco para o prejuízo visual grave e cegueira na infância, e frequentemente vem acompanhado por sequelas cerebrais, motoras e mentais.

Em estudos ${ }^{(7-9)}$ realizados na população de recém-nascidos (RN) do Recife, foi encontrada a catarata congênita como o principal comprometimento do cristalino, estrabismo como o principal transtorno dos músculos oculares e retinopatia da prematuridade como o transtorno da retina mais prevalente, em especial nos recém-nas- 
cidos prematuros (RNPT), sendo que pelo menos $60 \%$ destes comprometimentos visuais infantis são preveníveis ou tratáveis ${ }^{(10-11)}$.

Portanto, a identificação precoce de patologias visuais seguido de tratamento oportuno permitem aos lactentes uma maior integração com o seu meio, e consequentemente, um melhor DNPM ${ }^{(12)}$.

Segundo Gagliardo(13), os olhos são biologicamente determinados para a visão normal, mas é preciso experiências visuais durante a maturação do sistema visual, que possibilite a completa manifestação do potencial genético. Durante o "período de maturação", "a visão aperfeiçoa-se ou deteriora-se de acordo com a qualidade do estímulo visual, e por isso, é importante que nesta fase a retina receba informações claras e precisas"(14), pois a privação de estímulos visuais neste período pode levar a alterações anatômicas e funcionais irreversíveis ${ }^{(15)}$.

A American Academy of Ophthalmology (AAO) e a American Association for Pediatric Ophthalmology and Strabismus (AAPOS) recomendam que todas as crianças entre 6 e 12 meses passem por uma triagem visual, no entanto, aquelas que apresentarem risco de desenvolver problemas oftalmológicos, como as nascidas prematuramente, e as que tiverem história familiar de patologias oculares devem ser avaliadas ainda na maternidade ${ }^{(16-17)}$.

Assim, faz-se necessário que a triagem visual seja realizada no primeiro trimestre de vida do prematuro para detectar possíveis alterações visuomotoras, e desta forma, encaminhá-los ao oftalmologista e para intervenção precoce com equipe multidisciplinar, na qual se insere o terapeuta ocupacional ${ }^{(13,18)}$.

O diagnóstico precoce permite aos terapeutas ocupacionais iniciar programas de estimulação visual, visando o desenvolvimento visual, desenvolvimento global e orientação à família, contribuindo para a eficiência visual e evitando deficiências secundárias como comprometimentos socioafetivos, cognitivos e motores ${ }^{(18)}$.

Este estudo tem por objetivo caracterizar o comportamento visuomotor e o perfil socioeconômico e demográfico de RNPT, no primeiro mês de vida.

\section{MÉTODOS}

Tratou-se de um estudo descritivo, de corte seccional no $1^{\circ}$ mês de vida, aninhado a um estudo longitudinal no $1^{\circ}, 2^{\circ}$ e $3^{\circ}$ meses de vida do projeto de pesquisa, intitulado: "Comparação do comportamento visual no primeiro trimestre de vida de lactentes nascidos pré-termo em duas maternidades da cidade do Recife/PE".

A população do estudo foi selecionada entre RN prematuros nascidos na maternidade do Hospital das Clínicas da Universidade Federal de Pernambuco (HC-UFPE) no período de junho de 2007 a junho de 2008, que atenderam aos seguintes critérios de inclusão: pré-termo com idade gestacional compreendida entre 28 semanas completas e 36 semanas e 6 dias, pois o nascimento abaixo de 28 semanas aumenta o risco de apresentar retinopatia da prematuridade, podendo assim, o RNPT não responder adequadamente as provas da avaliação; de alta hospitalar ou internado, estar em condições clínicas favoráveis para realização da avaliação; e autorização dos responsáveis para sua participação no estudo. Foram excluídos os lactentes que apresentaram lesão neurológica, Apgar menor que 4 no $5^{\circ}$ minuto, já que indica asfixia grave ${ }^{(19)}$; malformação e/ou síndrome genética diagnosticada, infecção congênita confirmada e aqueles que não compareceram à avaliação pré-agendada para o primeiro mês de vida.

Dentre os 111 lactentes convidados, 52 (47\%) compareceram para a avaliação no primeiro mês de vida e foram excluídos 59 (53\%) por apresentarem intercorrências como: internação em Unidade de Terapia Intensiva Neonatal (UTIN) durante o período de avaliação, intercorrências neurológicas de qualquer natureza, óbito, falta a 1a avaliação e/ou cujos responsáveis desistiram voluntariamente em participar do estudo. Constituindo a amostra de 52 (47\%) RNPT com um mês de idade cronológica.
Os dados referentes às variáveis biológicas da criança (idade gestacional, peso ao nascer, sexo e Apgar), foram colhidos diretamente no prontuário dos lactentes. Já as variáveis maternas (idade, capacidade de leitura, escolaridade), as socioeconômicas e demográficas (renda familiar per capita, número de cômodos da residência, tipo de piso da residência, coabitação dos pais, trabalho paterno e bens de consumo) foram respondidas pelo responsável da criança, por meio de questionário contendo perguntas fechadas, coletados pela equipe de recrutamento.

O comportamento visuomotor dos RN foi avaliado por meio do Método de Avaliação da Conduta Visual de Lactentes ${ }^{(13)}$. O método é composto de 10 provas que qualificam as funções oculomotoras e apendiculares, como: fixação visual; contato de olho com o examinador; sorriso como resposta ao contato social; seguimento visual horizontal; seguimento visual vertical; exploração visual do ambiente; exploração visual da mão; aumento da movimentação de membros superiores ao visualizar o objeto; estender o braço na direção do objeto visualizado; sinais e sintomas oculares.

As avaliações foram realizadas no setor de Puericultura do HCUFPE por terapeutas ocupacionais, devidamente treinadas, utilizando como material um aro vermelho suspenso e o rosto do pesquisador que se referem ao instrumental do Método de Avaliação da Conduta Visual do Lactente ${ }^{(13)}$. Cada prova pode ser realizada em até três tentativas, tendo duração máxima de 10 a 20 minutos toda a avaliação. Cada lactente foi avaliado na presença de seu responsável, em sala apropriada, nas terças e sextas-feiras, considerando o primeiro mês de vida, o período compreendido entre 23 dias a 37 dias após o nascimento.

Foram realizadas adaptações com autorização da autora do Método, quanto à postura do RNPT durante a avaliação. Se o lactente apresentasse peso inferior a $2.200 \mathrm{~g}$ era posicionado em decúbito dorsal em cunha com suporte para os pés, quando seu peso era superior a $2.200 \mathrm{~g}$ o lactente era posicionado em decúbito dorsal sobre travesseiro anatômico. Estas adaptações foram realizadas para favorecer a estabilidade postural e para reduzir a chance de regurgitação do lactente, já que a inibição da atividade reflexa no RNPT depende da maturação do sistema nervoso central e a regurgitação é comum nos lactentes até o primeiro ano de vida, sendo maior quanto mais baixo peso for o recém-nascido ${ }^{(20-21)}$.

Os dados coletados foram digitados no software Epi-Info 2004 todos os instrumentos utilizados foram pré-codificados e checados, e posteriormente foi procedida à entrada dos dados no programa. A análise foi realizada por meio de frequência simples. Este estudo foi aprovado pelo Comitê de Ética em Pesquisa do HC - UFPE, sob o no 136, em dezembro de 2006.

\section{RESULTADOS}

No período de junho de 2007 a junho de 2008 foram recrutados 111 RN prematuros. Destes 53 foram excluídos, uma parte por não terem comparecido à primeira avaliação pré-agendada e 6 por responderem a algum outro critério de exclusão. A amostra constituída foi de 52 (47\%) RNPT com um mês de idade cronológica.

A análise identificou que $70 \%$ dos RN eram do sexo masculino, $87 \%$ prematuros moderado (> 33 semanas), com índice de Apgar maior que 7 no $5^{\circ}$ minuto (92\%) e peso ao nascer superior a $1.499 \mathrm{~g}$ (90\%). Com relação às famílias a maioria das mães (96\%) era adulto jovem, todas frequentaram a escola e sabiam ler e 55\% delas tinham 8 anos ou mais de estudo. Também possuíam bens de consumo diversificados como: fogão a gás, televisão, geladeira, rádio, aparelho de som, DVD e telefone celular.

Ainda em relação às variáveis socioeconômicas, (52\%) das famílias possuía renda mensal maior que um salário mínimo $(R \$ 380,00)$. A maioria das residências tinha de 4 a 6 moradores incluindo o RN (48\%), tendo como chefe de família o pai (63\%). As mães estavam desempregadas (62\%) e tinham em casa até 3 crianças menores de 5 anos incluindo o RN do estudo (100\%). A maioria das famílias vivia 
em boas condições demográficas residindo em casa de alvenaria (96\%), com piso de cimento/granito (71\%), teto de telha de barro/ amianto (75\%), possuindo mais de 4 cômodos (79\%), com canalização sanitária (96\%), água encanada dentro do quintal (77\%), coleta direta de lixo (94\%) e com energia elétrica (100\%).

Com relação ao desempenho visuomotor dos RN, estes apresentaram resposta positiva para as provas de coordenação oculomotora. Os sinais oculares como desvio, secreção e edema estiveram presentes em 19\% das crianças (Tabela 1).

\section{DISCUSSÃO}

Neste estudo foi possível verificar que a maioria das famílias apresentou boas condições socioeconômicas e demográficas, possuindo vários bens de consumo, estando acima da linha de pobreza, demonstrando as referidas condições como um fator de proteção para o desempenho funcional dos recém-nascidos. Na literatura, variáveis associadas à pobreza como a renda e o consumo de bens, quando deficitários pode prejudicar a harmonia dos pais e o bemestar do ambiente, podendo interferir na qualidade da relação familiar, desfavorecendo o desenvolvimento infantil ${ }^{(1,22)}$.

As mães entrevistadas tinham mais de 8 anos de estudo, bom nível de leitura e se concentravam na faixa etária de adultos jovens, mostrando que apresentavam um regular nível de compreensão e acesso a informações, facilitando uma interação adequada com o bebê. Martins et al. ${ }^{(2)}$ relatam que mães com maior escolaridade teriam mais acesso a informações sobre o desenvolvimento infantil, e desta maneira facilitariam a estimulação e o desempenho de novas habilidades em seus filhos ${ }^{(1)}$. Todas as famílias tinham até 3 crianças menores de 5 anos incluindo o RN do estudo. Este número reduzido de irmãos seria um fator protetor já que a literatura considera que famílias numerosas, com mais de quatro irmãos seriam menos estimuladoras e podendo levar a dificuldades no DNPM ${ }^{(1,22)}$.

Em todas as famílias do estudo, os pais viviam juntos podendo este ser considerado um fator de proteção, visto que a literatura aponta o suporte paterno como influenciador do afeto da mãe para com o filho, favorecendo a eficácia do cuidado materno ${ }^{(22)}$.

A maioria das habitações tinha 4 ou mais cômodos, fato este, facilitador do desenvolvimento infantil já que apresentam condições mais adequadas de iluminação, ventilação, espaço, temperatura e umidade, favorecendo a qualidade de vida ${ }^{(23)}$.

A maioria dos $\mathrm{RN}$ era prematuro moderado, tinha peso ao nascer superior a $1.499 \mathrm{~g}$, sendo considerada uma população de risco biológicos devido à prematuridade ${ }^{(22)}$.

Com relação às funções oculomotoras de fixação visual, contato de olho com o examinador, seguimento visual horizontal e exploração visual do ambiente, os RNPT apresentaram respostas positivas para estes comportamentos desde o $1^{\circ}$ mês de vida, assim como os $\mathrm{RN}$ a termo ${ }^{(13,24-25)}$. A menor frequência de respostas positivas para o comportamento de sorriso, está em concordância com a literatura, que descreve maior frequência desta função a partir do $2^{\circ}$ mês de vida ${ }^{(16,24-25)}$

Para Ribas ${ }^{(26)}$, o sorriso do bebê é uma atividade que está vinculada ao comportamento recíproco da mãe, ou seja, é necessário investigar mais profundamente se o comportamento de sorriso social destes lactentes teria relação apenas com a imaturidade neurológica, ou se existiria alguma influência do tempo de internação em UTIN, proporcionando pouca interação mãe-bebê.

A menor proporção de respostas positivas na função de seguimento visual vertical demonstra uma baixa coordenação oculomotora, o que corrobora com a literatura, tendo em vista que ao nascimento este comportamento está só iniciando, devido ao pequeno número de conexões sinápticas no córtex visual ao $1^{\circ}$ mês de vida ${ }^{(13,24-25)}$

Quanto aos comportamentos de exploração visual da mão, aumento da movimentação de membros superiores ao visualizar o objeto e estender o braço na direção do objeto visualizado foram funções não observadas nos RNPT nesta faixa etária. Tais achados estão de acordo
Tabela 1. Comportamento visual dos recém-nascidos prematuros no 10 mês de idade cronológica, na Maternidade do Hospital das Clínicas (HC/UFPE) Recife/PE. 2008

\begin{tabular}{|c|c|c|}
\hline \multirow[b]{2}{*}{ Variável } & \multicolumn{2}{|c|}{ Frequência $\mathbf{N}=\mathbf{2 0}$} \\
\hline & $\mathbf{n}$ & $\%$ \\
\hline \multicolumn{3}{|c|}{ Fixação visual } \\
\hline Sim & 50 & 96 \\
\hline Não & 2 & 4 \\
\hline \multicolumn{3}{|c|}{ Contato de olho com o examinador } \\
\hline Sim & 37 & 71 \\
\hline Não & 15 & 29 \\
\hline \multicolumn{3}{|c|}{ Sorriso como resposta ao contato social } \\
\hline Sim & 2 & 4 \\
\hline Não & 50 & 96 \\
\hline \multicolumn{3}{|c|}{ Sinergia oculocefalógira. Seguimento visual horizontal } \\
\hline Sim & 35 & 67 \\
\hline Não & 17 & 33 \\
\hline \multicolumn{3}{|c|}{ Sinergia oculocefalógira. Seguimento visual vertical } \\
\hline Sim & 11 & 21 \\
\hline Não & 41 & 79 \\
\hline \multicolumn{3}{|c|}{ Exploração visual do ambiente } \\
\hline Sim & 40 & 77 \\
\hline Não & 12 & 23 \\
\hline \multicolumn{3}{|c|}{ Exploração visual da mão } \\
\hline Sim & 0 & 0 \\
\hline Não & 52 & 100 \\
\hline \multicolumn{3}{|c|}{$\begin{array}{l}\text { Aumento da movimentação de } \\
\text { membros superiores }\end{array}$} \\
\hline Sim & 0 & 0 \\
\hline Não & 52 & 100 \\
\hline \multicolumn{3}{|c|}{ Estender o braço na direção do objeto visualizado } \\
\hline Sim & 0 & 0 \\
\hline Não & 52 & 100 \\
\hline \multicolumn{3}{|c|}{ Sinais oculares } \\
\hline Sim & 10 & 19 \\
\hline Não & 42 & 81 \\
\hline
\end{tabular}

com a literatura que descreve o início destas funções por volta do $2^{\circ}$ e $3^{\circ}$ meses de vida ${ }^{(13,24-25)}$.

As crianças com os sinais oculares detectados de ptose palpebral e secreção ocular foram encaminhadas ao serviço de Oftalmologia. No entanto, o lactente com desvio ocular foi reavaliado nos meses subsequentes sem encaminhamento já que o sinal apresentado é comum e pode ser fisiológico nos primeiros meses de vida ${ }^{(25)}$.

Com esta amostra foi possível identificar que o comportamento visuomotor dos RNPT no $1^{\circ}$ mês de vida, apresentou-se semeIhante ao desempenho do RN a termo, o que corrobora com a literatura pesquisada, mostrando que este comportamento recebe influências da maturação neuronal, e de fatores socioeconômicos e demográficos do ambiente em que vivem ${ }^{(24-25)}$. No entanto, seria interessante o acompanhamento destes RN para comparar o desenvolvimento visual dos mesmos, o que é fundamental para o planejamento de ações efetivas de prevenção e de reabilitação visual.

\section{CONCLUSÕES}

O Método de Avaliação do Comportamento Visual de Lactentes configurou-se como um instrumento adequado na avaliação da função visuomotora do RNPT, pois permitiu verificar como os RNPT usam a visão dentro do seu universo sensório-motor, por meio da observação clínica, característica básica do método.

Demonstrou que o desempenho visuomotor dos RNPT na faixa etária pesquisada é semelhante ao de lactentes a termo. Apontando 


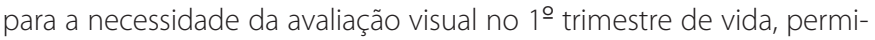
tindo a detecção precoce de alterações das funções visuais básicas, possibilitando o encaminhamento para diagnóstico e intervenção precoce em programa de estimulação visual, facilitando o desenvolvimento global da criança e o seu desempenho ocupacional de acordo com a realidade ambiental em que vive.

Observou-se que, o comportamento visual é uma habilidade que recebe influências multifatoriais, levando-nos a acreditar que o olhar clínico da intervenção terapêutica ocupacional deve estar voltado não apenas aos riscos biológicos da criança, mas para a influência que os fatores psicossociais exercem sobre o desempenho ocupacional da mesma.

Muito embora seja recomendada pela American Academy of Ophthalmology (AAO) e pela American Association for Pediatric Ophthalmology and Strabismus (AAPOS) a avaliação fundoscópica de prematuros com o oftalmoscópio binocular indireto não foi o objetivo deste trabalho. Porém, é muito importante e fazem-se necessários novos estudos, correlacionando os achados oftalmoscópicos com o comportamento visuomotor de RNPT.

\section{AGRADECIMENTOS}

Projeto financiado pelo Edital Universal do Conselho Nacional de Desenvolvimento Científico e Tecnológico (CNPq)/2006-2007.

\section{REFERÊNCIAS}

1. Mancini MC, Megale L, Brandão MB, Melo APP, Sampaio RF. Efeito moderador do risco social na relação entre risco biológico e desempenho funcional infantil. Rev Bras Saúde Matern Infant. 2004:4(1):25-34

2. Martins MFD, Costa JSD, Saforcada ET, Cunha MDC. Qualidade do ambiente e fatores associados: um estudo em crianças de Pelotas, Rio Grande do Sul, Brasil. Cad Saúde Pública = Rep Public Health. 2004;20(3):710-8.

3. Rugolo LMSS. Crescimento e desenvolvimento a longo prazo do prematuro extremo. J Pediatr (Rio J). 2005:81 (1 Supl):S101-10.

4. Barradas J, Fonseca A, Guimarães CLN, Lima GMS. A relação entre posicionamento do prematuro no Método Mãe-Canguru e desenvolvimento neuropsicomotor precoce. J Pediatr (Rio J). 2006;82(6):475-80

5. Figueiredo DV, Formiga CKMR, Tudella E. Aplicação de um programa de estimulação sensorial em bebês pré-termo em unidade de cuidados intermediários neonatais. Temas Desenvolv. 2003;12(71):15-22.

6. American Academy of Ophthalmology. Preferred Practice Patterns. Pediatric Eye Evaluations: Screeening and Comprehensive Ophthalmic Evaluation PPP - September 2007. [Internet]. [cited 2007 Set 7]. Available from: http://www.aao.org/ppp.
7. Albuquerque RC, Alves JGB. Afecções oculares prevalentes em crianças de baixa renda atendidas em um serviço oftalmológico na cidade do Recife - PE, Brasil. Arq Bras Oftalmol. 2003;66(6):831-4

8. Ventura LMO, Travassos S, Ventura AG, Trigueiro L, Marques S. Um programa baseado na triagem visual de recém-nascidos am maternidades: Fundação Altino Ventura/2000. Arq Bras Oftalmol. 2002;65(6):629-35.

9. Endriss D, Ventura LMVO, Diniz JR, Celino AC, Toscano J. Doenças oculares em neonatos Arq Bras Oftalmol. 2002;65(5):551-5.

10. Leal DB, Tavares SS, Ventura LO, Florencio T. Atendimento a portadores de visão subnormal: estudo retrospectivo de 317 casos. Arq Bras Oftalmol. 1995;58(6):439-42.

11. Brito PR, Veitzman S. Causas de cegueira e baixa visão em crianças. Arq Bras Oftalmol 2000; 63(1):49-54.

12. Regolin K, França L, Jimenez L, Sakamoto RH, Kamida NTS, Matheus KRM, et al. Avaliação da função visual em crianças com paralisia cerebral tipo tetraparesia e diparesia espástica: apresentação de um instrumento em terapia ocupacional. Temas Desenvolv. 2006; 15(85/86):24-9.

13. Gagliardo HGRG, Gonçalves VMG, Lima MCMP. Método para avaliação da conduta visual de lactentes. Arq Neuropsiquiatr. 2004;62(2A):300-6.

14. Faria e Sousa SJ. Fisiologia e desenvolvimento da visão. Medicina (Ribeirão Preto). 1997; 30(1):16-9.

15. Graziano RM, Leone CR. Problemas oftalmológicos mais frequentes e desenvolvimento visual do pré-termo extremo. J Peditr (Rio J). 2005:81(1 Supl):S95-100.

16. American Academy of Ophthalmology. American Association for Pediatric Ophthalmology and Strabismus. Vision Screening for Infants and Children [Internet]. American Academy of Ophthalmology. 2007 [cited 2007 Mar 10]. Available from: http://one.aao. org/printerfriendly. aspx?cid=0ad11e02-6a8b-437e-8d01-f45eb18bc0b6.

17. Ottar-Pfeifer W. When should children have their eyes checked? Insight. 2005;30(2):17-20; quiz 21-2

18. Montilha RCI, Nobre MIRS, Gagliardo HGR. Atuação terapêutico-ocupacional junto a pacientes com transtornos da visão. In: De Carlo MMR, Luzo MCM. Terapia ocupacional: reabilitação física e contextos hospitalares. São Paulo: Roca; 2004. p. 276-91.

19. Cunha AA, Fernandes DS, Melo PF, Guedes MH. Fatores associados à asfixia perinatal. Rev Bras Ginecol Obstet. 2004;26(10):799-805.

20. Olhweiler L, Silva AR, Rotta NT. Estudo dos reflexos primitivos em pacientes recém-nascidos pré-termo normais no primeiro ano de vida. Arq Neuropsiquiatr. 2005; 63(2A):294-7.

21. Costa AJF, Silva GAP, Gouveia PAC, Pereira Filho EM. Prevalência de refluxo gastroesofágico patológico em lactentes regurgitadores.J Pediatr (Rio J). 2004:80(4): 291-5.

22. Pilz EML, Schermann LB. Determinantes biológicos e ambientais no desenvolvimento neuropsicomotor em uma amostra de crianças de Canoas/RS. Ciênc Saúde Coletiva. 2007: 12(1):181-90.

23. Teixeira JC, Heller L. Fatores ambientais associados à desnutrição infantil em áreas de invasão Juiz de Fora, MG. Rev Bras Epidemiol. 2004;7(3):270-8.

24. Ruas TCB, Ravanini SG, Martinez CS, Gagliardo HR, Françoso MFC, Rim PHH. Avaliação do comportamento de lactentes no primeiro e segundo meses de vida. Rev Bras Crescimento Desenvolv Hum. 2006:16(3):1-8.

25. Gagliardo HGRG, Nobre MIRS. Intervenção precoce na criança com baixa visão. Rev Neurociências. 2001;9(1):16-9.

26. Ribas AFP, Moura MLS. Manifestações iniciais de trocas interativas mãe-bebê e suas transformações. Estud Psicol (Natal). 1999;4(2):273-88. 